\title{
Improved TV Algorithm Based on Adaptive Multiplier for Interference Hyperspectral Image Decomposition
}

\author{
Jia Wen, ${ }^{1}$ Jun Wu, ${ }^{1}$ Fang Zhang, ${ }^{1}$ Ran Wei, ${ }^{1}$ Xianglei Xing, ${ }^{2}$ and Cailing Wang ${ }^{3}$ \\ ${ }^{1}$ Tianjin Key Laboratory of Optoelectronic Detection Technology and Systems, School of Electronics and Information Engineering, \\ Tianjin Polytechnic University, Tianjin 300387, China \\ ${ }^{2}$ College of Automation, Harbin Engineering University, Harbin 150001, China \\ ${ }^{3}$ College of Computer Science, Xian Shiyou University, Xian 710065, China
}

Correspondence should be addressed to Jia Wen; 448680289@qq.com

Received 11 February 2016; Revised 23 April 2016; Accepted 28 April 2016

Academic Editor: Zhaolin Lu

Copyright (C) 2016 Jia Wen et al. This is an open access article distributed under the Creative Commons Attribution License, which permits unrestricted use, distribution, and reproduction in any medium, provided the original work is properly cited.

Interference Hyperspectral Images (IHI) data acquired by Interference Hyperspectral Imaging Spectrometer exhibit many vertical interference stripes. The above characteristics will affect the application of dictionary learning and compressed sensing theory used on IHI data. According to the special characteristics of IHI data, many algorithms are proposed to separate the interference stripes layers and the background layers of IHI data in 2015, but the interference stripes layers are still not clean enough and the ideal background layers without interference stripes are also difficult to be obtained. In this paper, an improved total variation (TV) algorithm based on adaptive multiplier is proposed for IHI data decomposition. The value of the Lagrange multiplier is adaptive according to the unidirectional characteristics of IHI data. The proposed algorithm is used on Large Spatially Modulated Interference Spectral (LSMIS) images and is proved to provide better experimental results than the current algorithms both visually and quantitatively.

\section{Introduction}

The technology of interference hyperspectral imaging [1-3] is very powerful in the field of remote sensing, which can get the spatial and spectral information of the interested targets, and has been widely used in many fields, such as meteorology, geology, environmental monitoring, and military. The interference hyperspectral spectrometer has been successfully equipped in the "Chang'E" lunar exploration satellite. Interference hyperspectral imaging has become the research focus in the recent years. IHI is a kind of threedimensional massive data, which has high resolution and will also lead to the difficulty on the storage and transmission on remote sensing. According to the special characteristics of IHI data, it is necessary to design efficient compression methods. The current compression methods for IHI include predictive algorithms [4-6], transform algorithms [7, 8], vector quantization algorithms [9], and data coding algorithms $[10,11]$.
Due to the special image principle, there are many position-fixed interference stripes exist in each frame of IHI data, which will seriously affect the results of IHI compression and reconstruction [8]. The inherent characteristics of IHI data will seriously impact the direct application of many traditional methods, such as the predictive coding and wavelet transform, which also cannot meet the precondition of the compressed sensing theory.

In 2015, morphological component analysis (MCA) and total variation (TV) are adopted to separate IHI data, respectively [12, 13]. A frame of IHI data is decomposed into an interference stripes layer and a background layer. But the results are not satisfied as there are still obvious vertical interference stripes in background layers in [12] and the interference stripes layers are still not clean enough in [13].

However, an improved TV algorithm based on adaptive multiplier is proposed in this paper. The expected decomposition result is to obtain background layers without vertical interference stripes, and the interference stripes layers are 


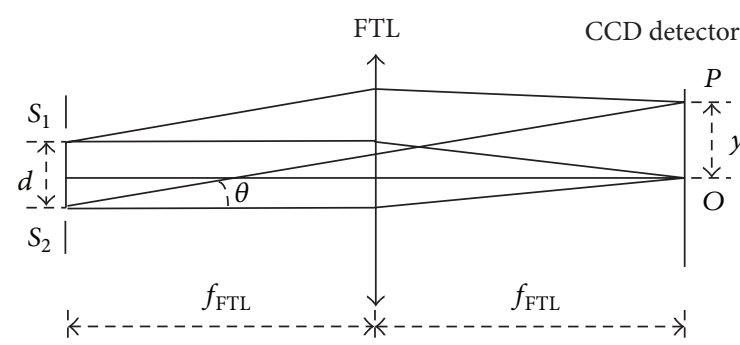

FIGURE 1: The sketch map of the interference hyperspectral spectrometer.

clean enough without any residual background pixels, which is the basic idea of the proposed method. The value of the Lagrange multiplier is adaptive according to the unidirectional characteristics of IHI data; that is, the horizontal variations of background layers and the vertical variations of interference stripes layers should be small enough, and the optimal value of the Lagrange multiplier will be helpful for obtaining the optimal solution.

The imaging principle and characteristics of IHI will be introduced in the next section. The TV algorithm will be introduced in Section 3. An improved TV algorithm based on adaptive multiplier will be proposed in Section 4 . Experiments and analysis will be given in Section 5 and the conclusion will be given in Section 6 .

\section{Imaging Principle and Characteristics of IHI}

Figure 1 shows the equivalent optical path in lateral shearing interferometer. $d$ is the distance between $S_{1}$ and $S_{2}$, which are the two separated rays by the light from a ground point. In the interferometer, Fourier transform lens (FTL) is the main imaging equipment. The Optical Path Difference (OPD) of point $O$ in Charge Coupled Device (CCD) is zero.

The OPD of point $P$ on the CCD detector is

$$
x=d \sin \theta=\frac{y d}{f_{\mathrm{FTL}}}
$$

where $f_{\mathrm{FTL}}$ is the focus of Fourier lens.

According to the theory of Fourier transform [14], the interference curve can be expressed as

$$
\begin{aligned}
I(x) & =\int_{k_{\min }}^{k_{\max }} B(k) e^{j 2 \pi k x} d k \\
& =\int_{k_{\min }}^{k_{\max }} B(k) e^{j 2 \pi k\left(y d / f_{\mathrm{FTL}}\right)} d k,
\end{aligned}
$$

in which $B(k)$ is the spectral distribution of source, $k_{\max }$ and $k_{\min }$ are the maximum and minimum of wavenumber, respectively, and $x$ represents OPD of this interference curve. Because $B(k)$ is a real and even function, its Fourier transform

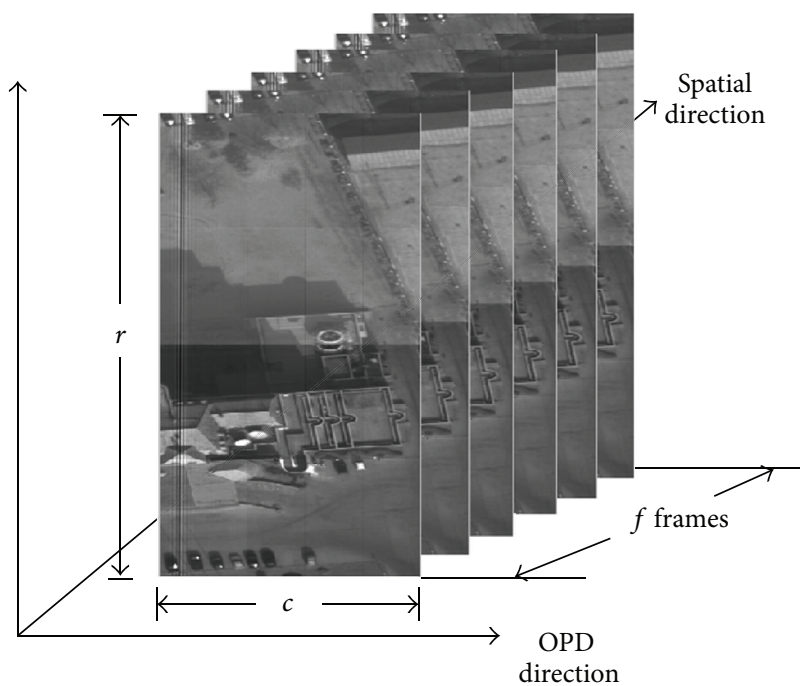

FIgURE 2: Three-dimensional LASIS IHI data.

must be a real and even function too [14]. So formula (2) equals

$$
\begin{aligned}
I(x) & =\int_{k_{\min }}^{k_{\max }} B(k) \cos (2 \pi k x) d k \\
& =\int_{k_{\min }}^{k_{\max }} B(k) \cos \left(2 \pi k y \frac{d}{f_{\mathrm{FTL}}}\right) d k .
\end{aligned}
$$

The spectrum curve can be obtained by inverse Fourier transform of the interference curve according to the basic Fourier transform relationship. The inverse Fourier transform of formula (2) is

$$
\begin{aligned}
B(k) & =\int_{0}^{\delta_{m}} I(x) e^{-j 2 \pi k x} d x \\
& =\int_{0}^{\delta_{m}} I(x) e^{-j 2 \pi k\left(y d / f_{\mathrm{FTL}}\right)} d x .
\end{aligned}
$$

$\delta_{m}$ is the maximum OPD. The corresponding cosine transform is

$$
\begin{aligned}
B(k) & =\int_{0}^{\delta_{m}} I(x) \cos (2 \pi k x) d x \\
& =\int_{0}^{\delta_{m}} I(x) \cos \left(2 \pi k y \frac{d}{f_{\mathrm{FTL}}}\right) d x .
\end{aligned}
$$

Figure 2 shows the sketch map of three-dimensional IHI data produced by LASIS [1-3].

The main characteristics of IHI data are as follows.

First, IHI is not directly imaging of light, so there are obvious vertical interference stripes in each frame of IHI.

Second, IHI is three-dimensional data formed through push-broom technique, so the background of IHI has horizontal shift between frames.

To get better result when the dictionary learning and compressed sensing algorithms are applied on IHI data, TV algorithm [15-18] is adopted to separate the interference stripes from the background in [13]. 


$$
\begin{aligned}
& \text { Steps of the proposed method: } \\
& \text { Input: IHI data } X, \beta \in[\text { start_value, end_value }] \text {. } \\
& \qquad X_{B}=X ; \\
& \qquad \begin{array}{c}
O p t_{-} \beta=\mathrm{GA}(\beta, \operatorname{FitFun}(\beta)) \\
X_{B}=\underset{X_{B}}{\arg \min }\left\{\mathrm{TV}_{y}\left(X-X_{B}\right)+O p t_{-} \beta \cdot \mathrm{TV}_{x}\left(X_{B}\right)\right\} ; \\
X_{I}=X-X_{B} ;
\end{array}
\end{aligned}
$$

Output: $X_{B}, X_{I}$

Steps of the FitFun of Genetic Algorithm

Input: $\beta \in[$ start_value, end_value $], \eta, X$.

$$
\begin{aligned}
& \text { for iter = } 1 \text { : ini_num } \\
& \qquad X_{B}=X_{B}-\eta \frac{\partial\left\{\int_{x, y \in X} \sqrt{\left(\partial\left(X(x, y)-X_{B}(x, y)\right) / \partial y\right)^{2}}+\beta \int_{x, y \in X} \sqrt{\left(\partial X_{B}(x, y) / \partial x\right)^{2}}\right\}}{\partial X_{B}} ; \\
& \text { end }
\end{aligned}
$$

$$
\text { Fitness }=\operatorname{abs}\left(\operatorname{TV}_{x}\left(X_{B}\right)\right)+\operatorname{abs}\left(\operatorname{TV}_{y}\left(X-X_{B}\right)\right)
$$

Output: Fitness

Algorithm 1

\section{TV Algorithm for IHI Data Decomposition}

The effect of interference stripes in one frame of IHI data $X$ is assumed to be an additive layer $X_{I}$, while the background pixels of IHI data are assumed to be in another layer $X_{B}$. So the decomposition process can be formulated as follows:

$$
X=X_{I}+X_{B}
$$

TV algorithm is proposed for IHI data decomposition in 2015 [13]. As the interference stripes in $X_{I}$ are vertical, so their variations are mainly concentrated along the $x$-axis. In mathematical words, most pixels of the stripes have the property as follows:

$$
\operatorname{TV}_{y}\left(X_{I}\right) \ll \operatorname{TV}_{x}\left(X_{I}\right)
$$

where $\mathrm{TV}_{x}$ and $\mathrm{TV}_{y}$ are horizontal and vertical total variations, respectively. The formula (7) can also be written as [1518]

$$
\int_{x, y \in X} \sqrt{\left(\frac{\partial X_{I}(x, y)}{\partial y}\right)^{2}} \ll \int_{x, y \in X} \sqrt{\left(\frac{\partial X_{I}(x, y)}{\partial x}\right)^{2}} .
$$

So the following function is proposed in [19] for IHI data decomposition:

$$
X_{B}=\underset{X_{B}}{\arg \min }\left\{\mathrm{TV}_{y}\left(X-X_{B}\right)+\beta \mathrm{TV}_{x}\left(X_{B}\right)\right\},
$$

where $\beta$ is the Lagrange multiplier which quantifies the degree of smoothness across the $x$-axis. The formula (9) can also be written as

$$
\begin{aligned}
& X_{B} \underset{X_{B}}{\arg \min }\left\{\int_{x, y \in X} \sqrt{\left(\frac{\partial\left(X(x, y)-X_{B}(x, y)\right)}{\partial y}\right)^{2}}\right. \\
& \left.+\beta \int_{x, y \in X} \sqrt{\left(\frac{\partial X_{B}(x, y)}{\partial x}\right)^{2}}\right\} .
\end{aligned}
$$

The traditional gradient descent algorithm is adopted to get the optimal result of $X_{B}$ in the formula (10) in [19], and the optimal result of $X_{I}$ will also be obtained through the difference between $X$ and $X_{B}$.

\section{Proposed TV Algorithm Based on Adaptive Multiplier}

Although many methods [12, 13] have been proposed in order to separate the interference stripes layers from the background layers, the decomposition results are still not completely satisfied. Vertical interference stripes still exist in background layers obviously in [12] and the interference stripes layers still contain the background pixels in [13].

In this paper, an improved TV algorithm based on adaptive multiplier is proposed for IHI data decomposition. The Lagrange multiplier $\beta$ plays a key role in the process of decomposition. If $\beta$ is too small, there will be residual stripes in the background layers $X_{B}$, while a large value of $\beta$ will lead to oversmoothing. So the choice of an optimal Lagrange multiplier is very important in TV algorithm. 


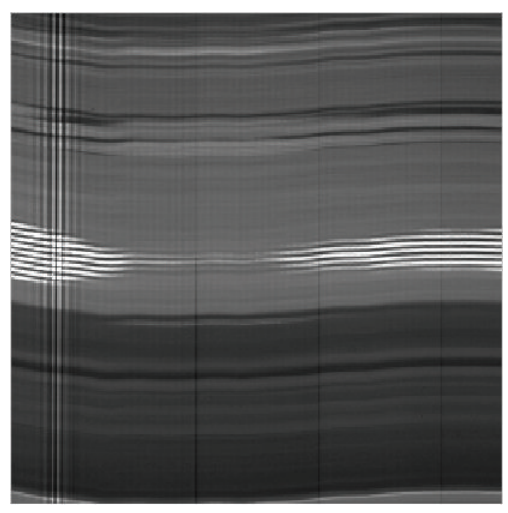

(a)

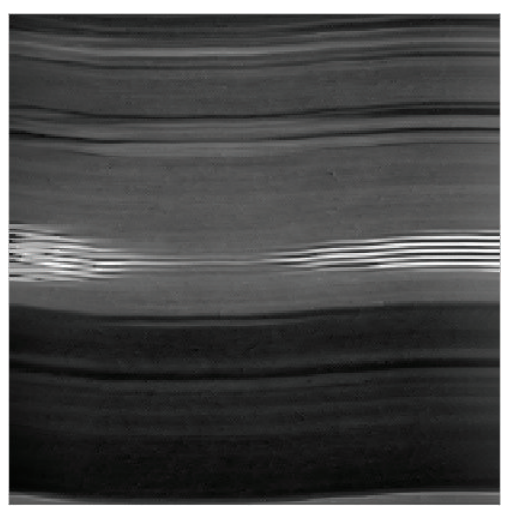

(d)

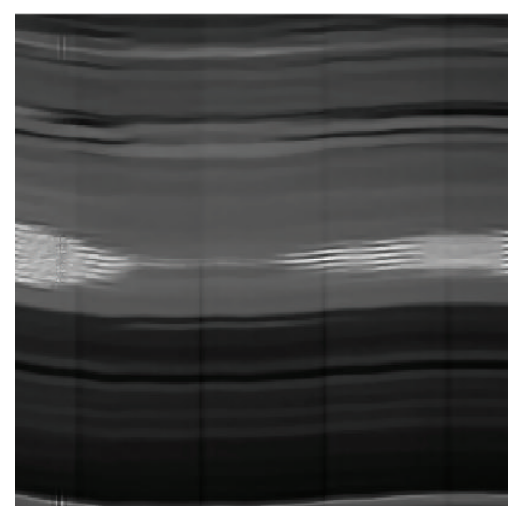

(b)

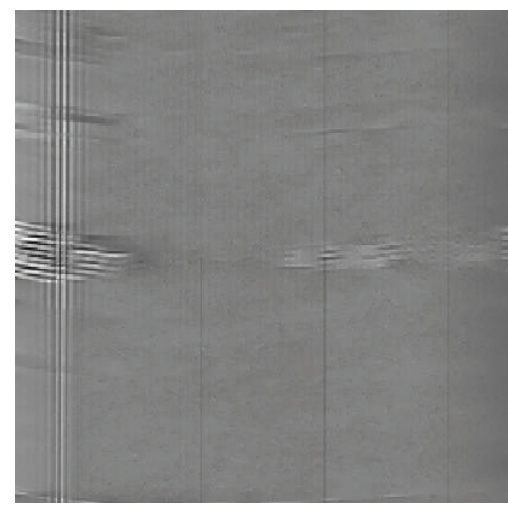

(e)

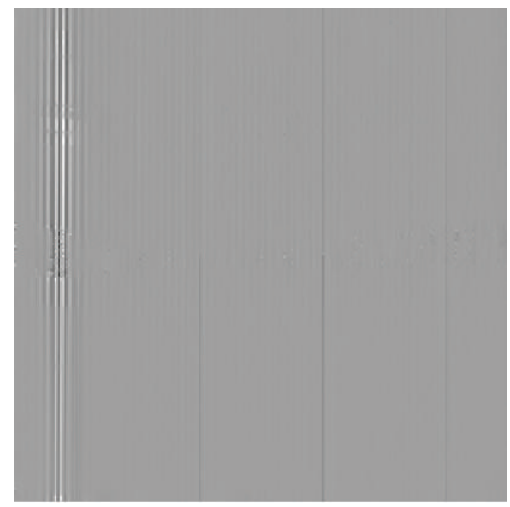

(g)

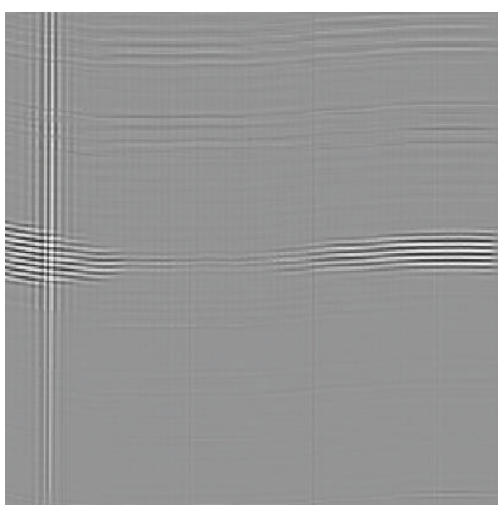

(c)

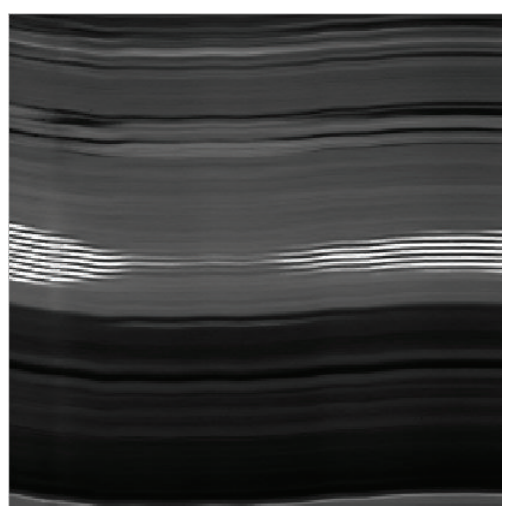

(f) FIGURE 3: Experimental results of LSMIS data 1. (a) Original LSMIS data 1. (b) Background layer produced by MCA. (c) Interference stripes
layer produced by MCA. (d) Background layer produced by IMT. (e) Interference stripes layer produced by IMT. (f) Background layer produced by the proposed method. (g) Interference stripes layer produced by the proposed method.

The basic idea of the proposed algorithm is based on the special characteristics of IHI data. For the ideal result of decomposition, $X_{B}$ should only contain the background pixels without any vertical stripes, as the special imaging principle of IHI data; the background layers should have unidirectional property because the values of most pixels are almost the same in the horizontal direction, while $X_{I}$ should only contain the vertical interference stripes without any background pixels, which should have unidirectional property in vertical direction. As $X_{B}$ and $X_{I}$ should have unidirectional property in horizontal and vertical directions, respectively, the value of Lagrange multiplier $\beta$ should be variant depending on the horizontal and vertical total variations of the layers. The improved TV algorithm based on adaptive multiplier is proposed as in Algorithm 1.

Genetic Algorithm (GA) [20] is adopted in the proposed algorithm for calculating the optimal Lagrange multiplier $\beta$ and making it adaptive for different frames of IHI data. 


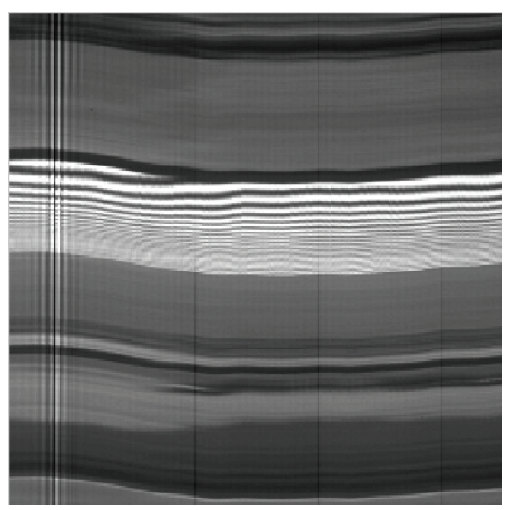

(a)

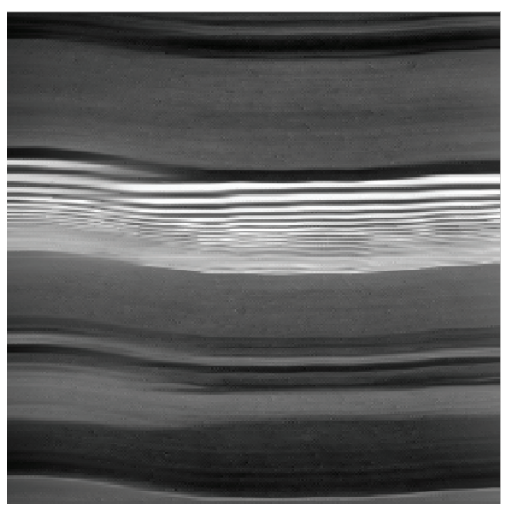

(d)

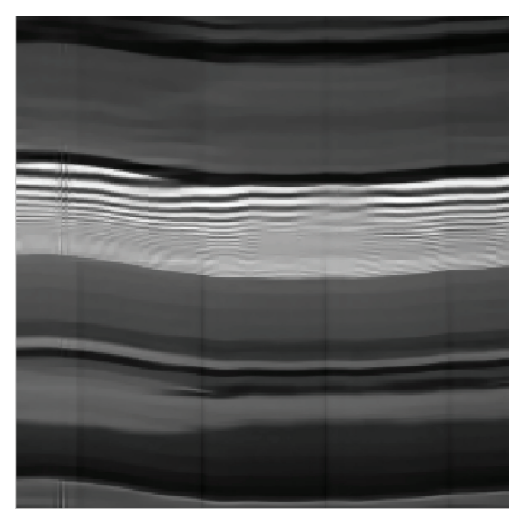

(b)

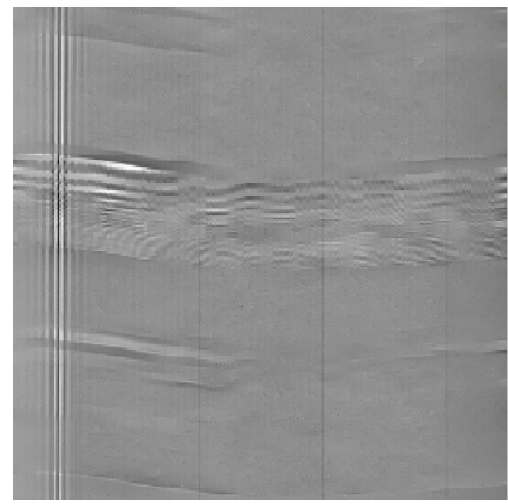

(e)

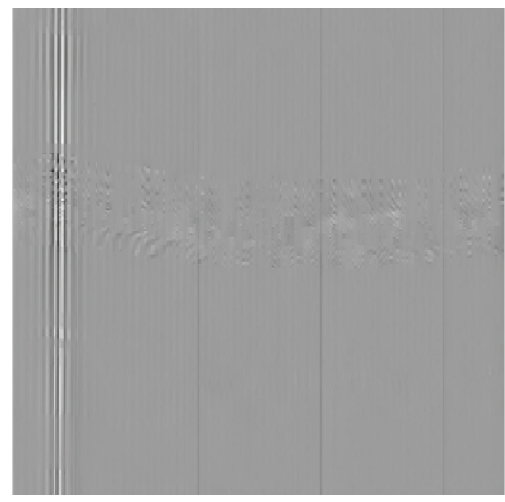

(g)

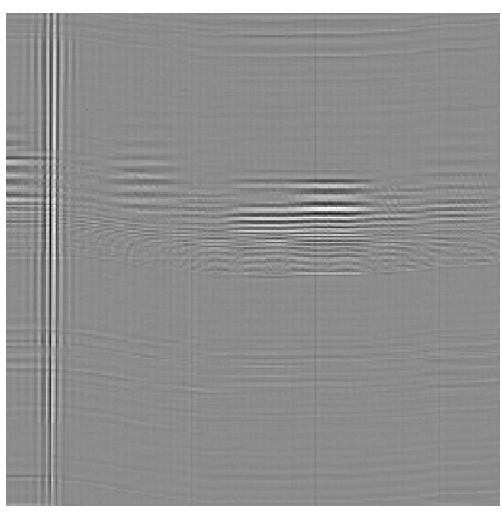

(c)
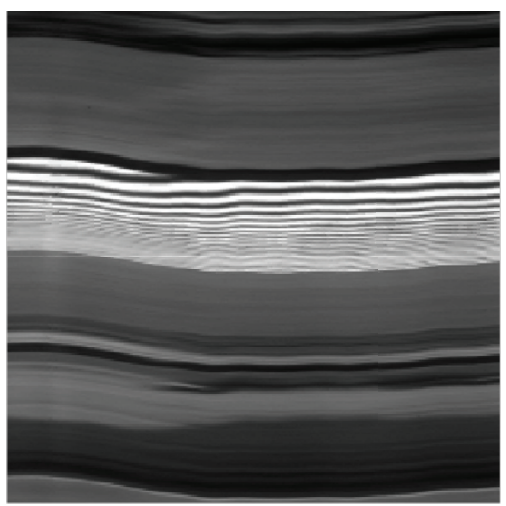

(f)

FIGURE 4: Experimental results of LSMIS data 2. (a) Original LSMIS data 1. (b) Background layer produced by MCA. (c) Interference stripes layer produced by MCA. (d) Background layer produced by IMT. (e) Interference stripes layer produced by IMT. (f) Background layer produced by the proposed method. (g) Interference stripes layer produced by the proposed method.

In ideal state, no interference stripes will exist in $X_{B}$, and only interference stripes will exist in $X_{I}$, so formula $(*)$ in Algorithm 1 is used for the fitness function of GA, which can keep the final outputs $X_{B}$ and $X_{I}$ having the minimum of horizontal and vertical total variations, respectively.

\section{Experiments and Analysis}

Three groups of LSMIS IHI data will be chosen for experiment. The LSMIS data is 12 bytes' image of size $256 \times 256$. Compared with the novel methods $[12,13]$, the proposed method will be used for LSMIS data decomposition. The start_value, end_value, $\eta$, and ini_num are chosen to be 0,3 , 0.2 , and 10 in the experiment, respectively. For LSMIS data $1-3$, values of the optimal multiplier calculated by GA are $0.6273,0.3963$, and 1.4851, respectively. Experimental results for visual comparisons are shown in Figures 3-5, and the quantitative results for comparisons are shown in Tables 1 and 2.

In Figures 3-5, the background layers produced by IMCA, IMT, and the proposed method are shown in (b), (d), and (f), respectively. The interference stripes layers produced 


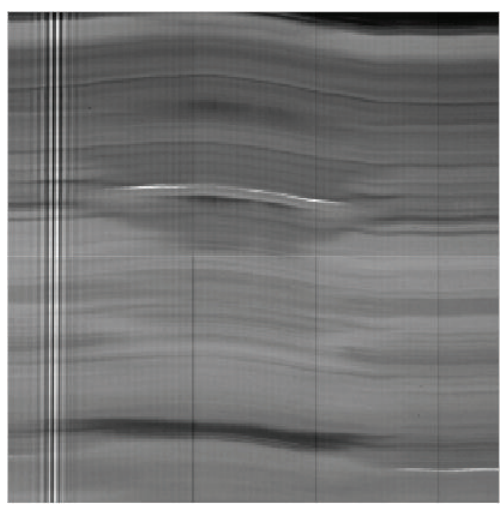

(a)

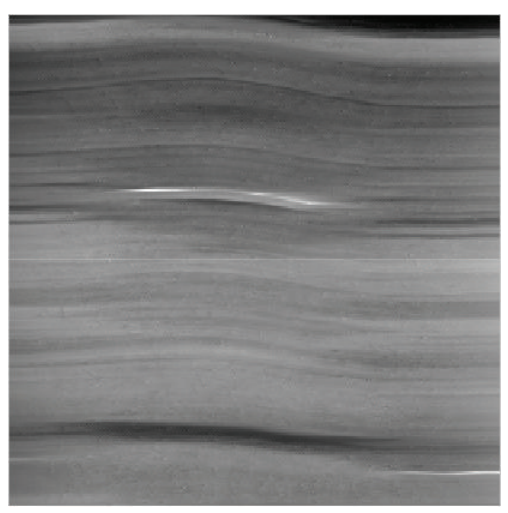

(d)

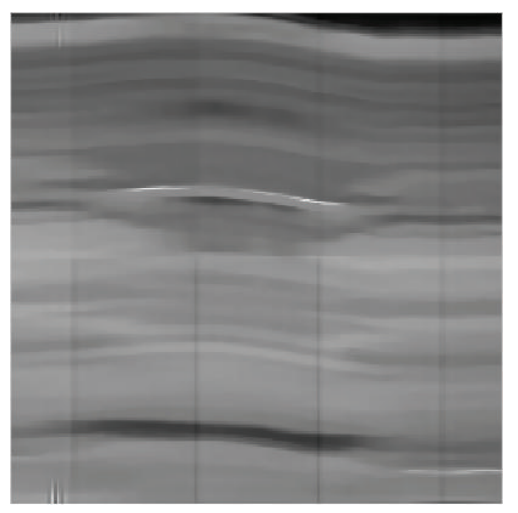

(b)

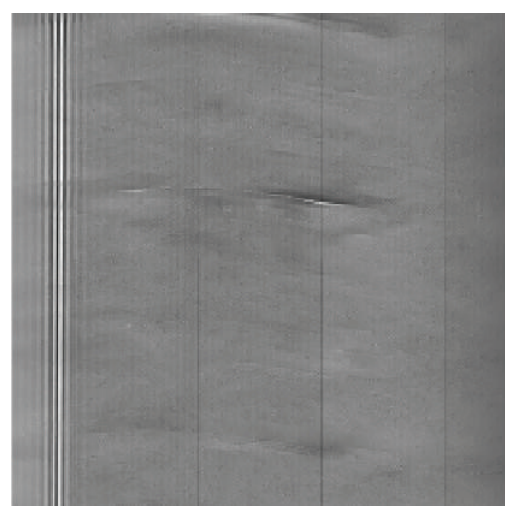

(e)

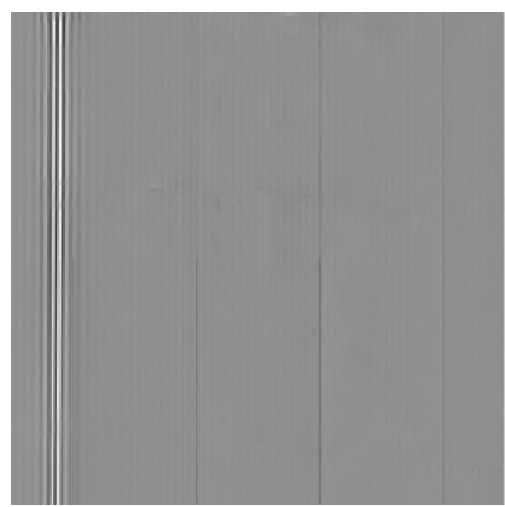

(g)

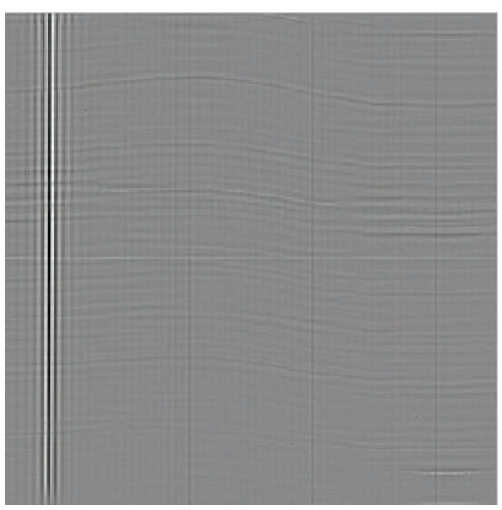

(c)

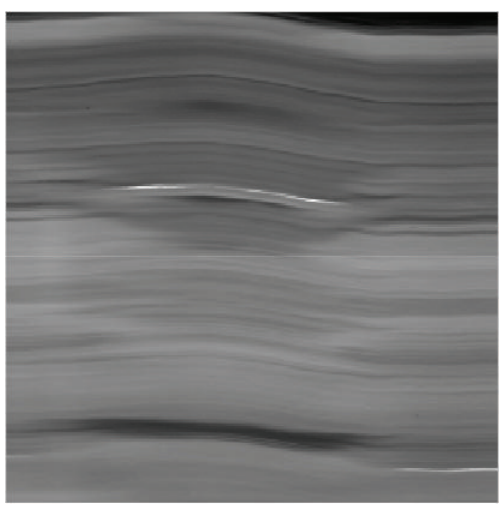

(f)

FIGURE 5: Experimental results of LSMIS data 3. (a) Original LSMIS data 1. (b) Background layer produced by MCA. (c) Interference stripes layer produced by MCA. (d) Background layer produced by IMT. (e) Interference stripes layer produced by IMT. (f) Background layer produced by the proposed method. (g) Interference stripes layer produced by the proposed method.

TABLE 1: Horizontal total variations of interference stripes part in $X_{B}$.

\begin{tabular}{lccc}
\hline & LSMIS data 1 & LSMIS data 2 & LSMIS data 3 \\
\hline IMCA [12] & $1.4236 e+05$ & $1.2562 e+05$ & $9.3652 e+04$ \\
IMT [13] & $1.3963 e+05$ & $1.1686 e+05$ & $9.2184 e+04$ \\
Proposed & $1.3285 e+05$ & $1.0716 e+05$ & $3.4778 e+04$ \\
\hline
\end{tabular}

by IMCA, IMT, and the proposed method are shown in (c), (e), and (g), respectively. We can clearly see that the
TABLE 2: Vertical total variations in $X_{I}$.

\begin{tabular}{lccc}
\hline & LSMIS data 1 & LSMIS data 2 & LSMIS data 3 \\
\hline IMCA [12] & $3.2000 e+06$ & $3.2516 e+06$ & $1.3611 e+06$ \\
IMT [13] & $1.8571 e+06$ & $2.4897 e+06$ & $1.1145 e+06$ \\
Proposed & $3.0733 e+05$ & $4.0003 e+05$ & $3.3235 e+05$ \\
\hline
\end{tabular}

residual stripes still exist at the top and bottom of interference stripes parts in (b), while in (d) and (f) the stripes are 
almost removed. And as there are few residual background pixels in the interference stripes layers produced by the proposed method, the layers in (g) are relatively much cleaner compared with (c) and (e). And as the results shown in Tables 1 and 2, the proposed method can obtain $X_{B}$ and $X_{I}$ with smaller total variations in horizontal and vertical directions, respectively. The reason of the experimental results is that in the regularization process of the proposed method, the unidirectional characteristics of ideal background layers and interference layers are both taken into account.

\section{Conclusion and Prospect}

IHI data has different characteristics from natural images due to the special imaging principle, which will affect the direct application of dictionary learning and compressed sensing algorithms. An improved TV algorithm based on adaptive multiplier is proposed for IHI data decomposition, which will separate IHI data into two different layers and make them sparsely represented and meet the precondition of compressed sensing. Compared with other decomposition methods for IHI data, the proposed method can obtain background layers and interference stripes layers with smaller total variations in horizontal and vertical directions, respectively.

\section{Competing Interests}

The authors declare that they have no competing interests.

\section{Acknowledgments}

This work was supported by the National Natural Science Foundation of China (Grant no. 61401439), project of Tianjin Application Foundation, and advanced technology research program (15JCYBJC16600).

\section{References}

[1] A. R. Harvey and D. W. Fletcher-Holmes, "Birestripent Fouriertransform imaging spectrometer," Optics Express, vol. 12, no. 22, pp. 5368-5374, 2004.

[2] A. Barducci, V. De Cosmo, P. Marcoionni, and I. Pippi, "ALISEO: a new stationary imaging interferometer," in Imaging Spectrometry X, vol. 5546 of Proceedings of SPIE, pp. 262-270, Denver, Colo, USA, 2004.

[3] B. Xiangli, Z. Gao, B. An, and B. Zhao, "Static imaging Fourier transform spectrometer," in Proceedings of the Conference on Hyperspectral Remote Sensing and Application, vol. 3502 of Proceedings of SPIE, pp. 30-34, September 1998.

[4] J. Wen, C. Ma, and P. Shui, "An adaptive OPD and dislocation prediction used characteristic of interference pattern for interference hyperspectral image compression," Optics Communications, vol. 284, no. 20, pp. 4903-4909, 2011.

[5] X.-L. Tu, M. Huang, Q.-B. Lü, J.-W. Wang, and L.-L. Pei, "Interference hyperspectral data compression based on spectral classification and local DPCM," Spectroscopy and Spectral Analysis, vol. 33, no. 5, pp. 1401-1405, 2013.
[6] J. Wu and J. Xu, "Clustered DPCM with removing noise spectra for the lossless compression of hyperspectral images," in Proceedings of the 8th Symposium on Multispectral Image Processing and Pattern Recognition (MIPPR '13), vol. 8917, Multispectral, October 2013.

[7] J. Wen, C. Ma, J. Zhao, and C. Wang, "An adaptive wavelet transformation used on interference hyperspectral image compression," Journal of Harbin Institute of Technology, vol. 46, no. 7, pp. 112-117, 2014.

[8] L.-M. Du, J. Li, G. Jin, H.-B. Gao, L.-X. Jin, and K. Zhang, "Compression of interference hyperspectral image based on FHALS-NTD," Spectroscopy and Spectral Analysis, vol. 32, no. 11, pp. 3155-3160, 2012.

[9] J. Wen, C. Ma, and J. Zhao, "FIVQ algorithm for interference hyper-spectral image compression," Optics Communications, vol. 322, pp. 97-104, 2014.

[10] D.-M. Ma, C.-W. Ma, and Y.-L. Bai, "An algorithm of 3DSPIHT for LASIS hyperspectral image compression based on BOI," Opto-Electronic Engineering, vol. 38, no. 3, pp. 125-130, 2011.

[11] D. Ma, C. Ma, and Y. Bai, "Modified listless 3DSPITH with ROI for hyperspectral image compression," Journal of Applied Optics, vol. 32, no. 3, pp. 446-451, 2011.

[12] J. Wen, J. Zhao, and C. Wang, "Improved morphological component analysis for interference hyperspectral image decomposition," Computers and Electrical Engineering, vol. 46, pp. 394402, 2015.

[13] J. Wen, J. Zhao, and W. Cailing, "Improved MCA-TV algorithm for interference hyperspectral image decomposition," Optics and Lasers in Engineering, vol. 75, pp. 81-87, 2015.

[14] J. W. Goodman, Introduction to Fourier Optics, Roberts \& Company, 2005.

[15] G. Zhou, H. Fang, L. Yan, T. Zhang, and J. Hu, "Removal of stripe noise with spatially adaptive unidirectional total variation," Optik, vol. 125, no. 12, pp. 2756-2762, 2014.

[16] L. Yan, H. Fang, and S. Zhong, "Blind image deconvolution with spatially adaptive total variation regularization," Optics Letters, vol. 37, no. 14, pp. 2778-2780, 2012.

[17] M. Bouali and S. Ladjal, “Toward optimal destriping of MODIS data using a unidirectional variational model," IEEE Transactions on Geoscience and Remote Sensing, vol. 49, no. 8, pp. 29242935, 2011.

[18] W. Hu, Y. Xie, L. Li, and W. Zhang, "A total variation based nonrigid image registration by combining parametric and nonparametric transformation models," Neurocomputing, vol. 144, pp. 222-237, 2014.

[19] M. Bouali and S. Ladjal, “Toward Optimal destriping of MODIS data using a unidirectional variational model," IEEE Transactions on Geoscience and Remote Sensing, vol. 49, no. 8, pp. 29242935, 2011.

[20] A. Bartoli, A. De Lorenzo, E. Medvet, and F. Tarlao, "Playing regex golf with genetic programming," in Proceedings of the 16th Genetic and Evolutionary Computation Conference (GECCO '14), pp. 1063-1069, ACM, Vancouver, Canada, July 2014. 

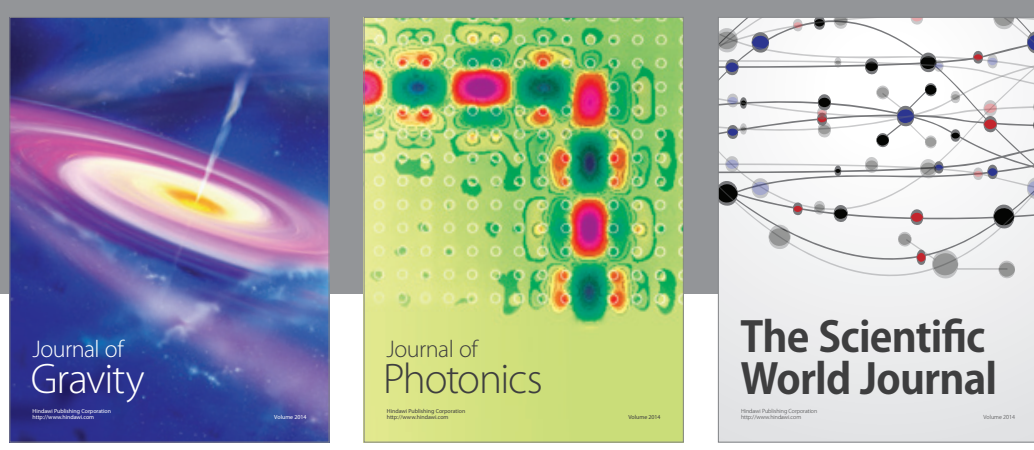

The Scientific World Journal
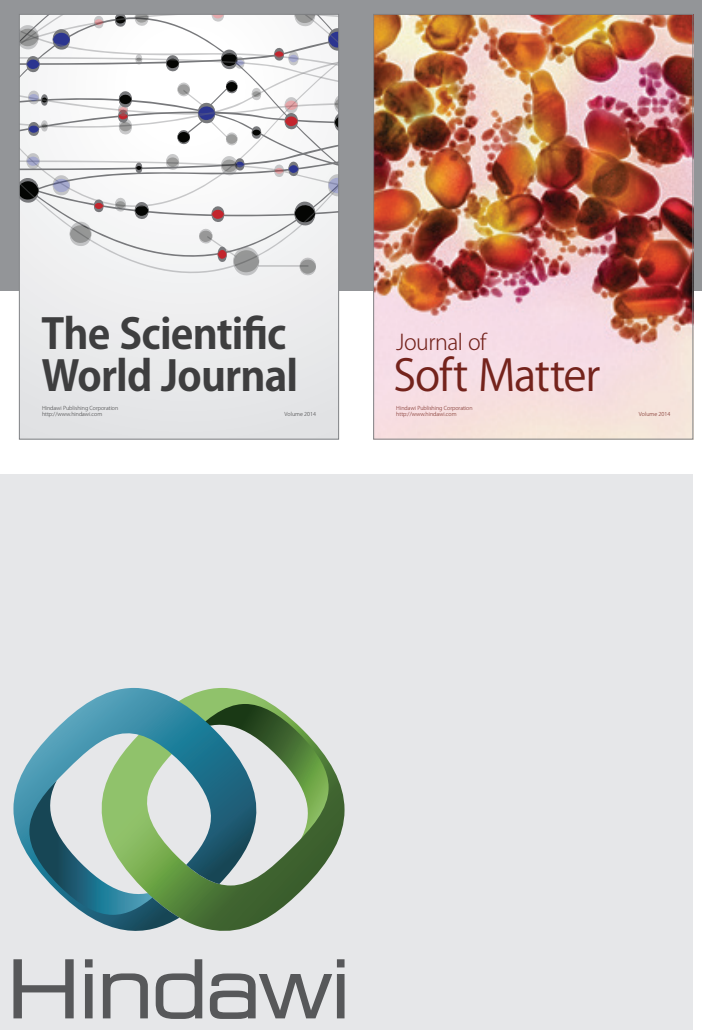

Submit your manuscripts at

http://www.hindawi.com

nternational Journal of

Statistical Mechanics
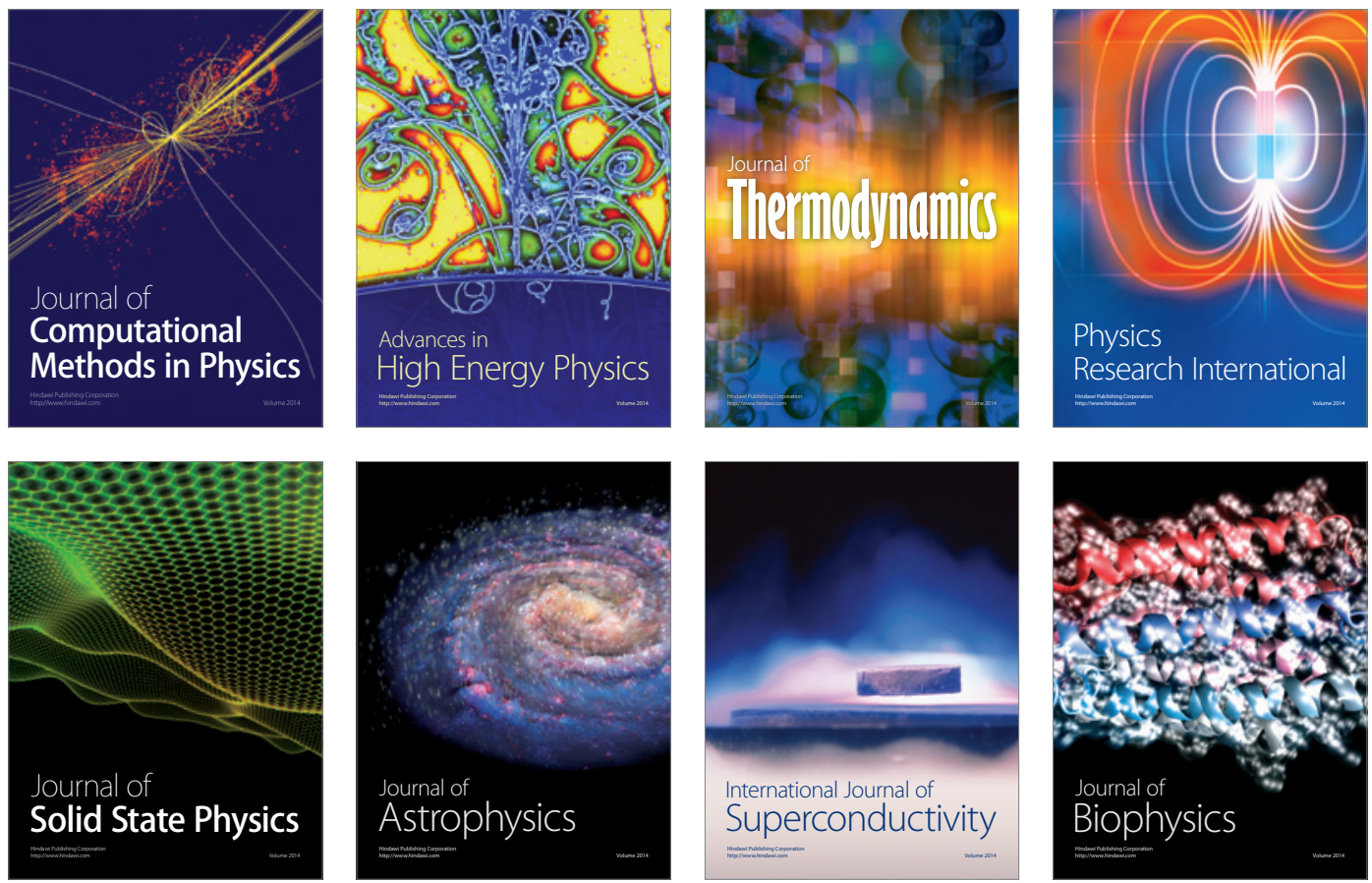
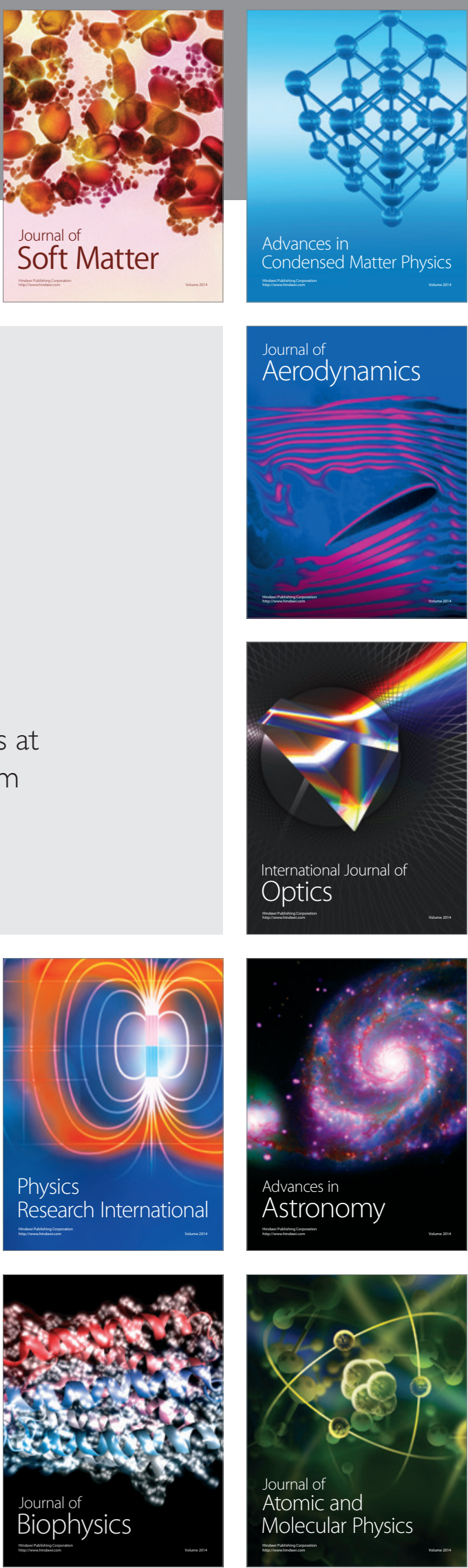